\title{
The Lockman Hole Project: A Multi-frequency Study of the Faint Radio Population down to LOFAR bands
}

\author{
G. Guglielmino ${ }^{1}$, I. Prandoni ${ }^{1}$, R. Morganti ${ }^{2,3}$, G. Heald ${ }^{2}$, \\ E. Mahony ${ }^{2}$ and I. van Bemmel ${ }^{2}$ \\ ${ }^{1}$ INAF-Institute of Radioastronomy, Bologna, Italy \\ ${ }^{2}$ ASTRON, the Netherlands Institute of Radio Astronomy, Dwingeloo, The Netherlands \\ ${ }^{3}$ Kapteyn Astronomical Institute, University of Groningen, Groningen, The Netherlands
}

\begin{abstract}
We are performing a multi-frequency radio analysis of a well-known deep field: the Lockman Hole, which is one of the best studied sky regions in different wavebands. This will provide us with important complementary data (for example redshifts) to the radio data, allowing us to characterize the physical and evolutionary properties of the various classes of sources composing the faint radio population. LOFAR imaging of the Lockman Hole can play an important role in this project, allowing, for the very first time, to observe the sub-mJy source population at very low frequencies $(30-200 \mathrm{MHz})$, where self-absorption phenomena are expected to be very important. Here we present some preliminary results.
\end{abstract}

\section{Background}

Multi-wavelength studies of deep radio fields show that the sub-mJy population has a composite nature. Star-forming galaxies dominate at $\mu \mathrm{Jy}$ levels, while radio loud $(\mathrm{RL})$ AGNs are the dominant component at flux densities $>0.5-1 \mathrm{mJy}$ (e.g. Prandoni et al.

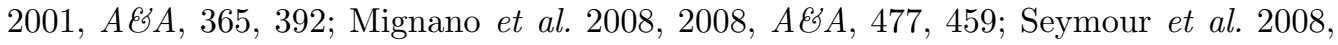
MNRAS, 386, 16 95; Smolcic et al. 2008, ApJS, 177, 14). Recently, a new class of sub$\mathrm{mJy}$ radio sources has been revealed at flux densities $<0.5 \mathrm{mJy}$, identified with radio quiet (RQ) AGNs (e.g. Padovani et al. 2009, ApJ, 694, 235; Bonzini et al. 2012, ApJS, 203, 15). A better understanding of the physical and evolutionary properties of such low/intermediate power AGNs may have important implications on the determination of the black-hole-accretion history of the Universe; and, more generally, would allow us a better understanding of the triggering mechanisms of AGN radio activity. Multiwavelength information and radio spectral indices may help to constrain the ongoing processes in such faint radio sources, and may be fundamental in understanding possible links to the bolometric emission of the host galaxies.

\section{WSRT Observations at $1.4 \mathrm{GHz}$ and $325 \mathrm{MHz}$}

The Lockman Hole (LH) was observed with the WSRT at $1.4 \mathrm{GHz}$ and $325 \mathrm{MHz}$ (Guglielmino et al. 2013a,b, in prep.). The $1.4 \mathrm{GHz}$ mosaic observations cover an area of 6.6 square degrees. The rms sensitivity is approximately uniform over the central 2 sq. degr. region, with a value of about $11 \mu \mathrm{Jy}$. The extracted catalog lists 6002 sources. This is one of the largest $\mu \mathrm{Jy}$ samples available so far. The image obtained at $345 \mathrm{MHz}$ is shallower, being confusion-limited at a level of rms $\sim 0.6-0.7 \mathrm{mJy}$. Nevertheless such data can be useful to study the radio spectral behaviour of the mJy radio sources in the Lockman Hole field. 


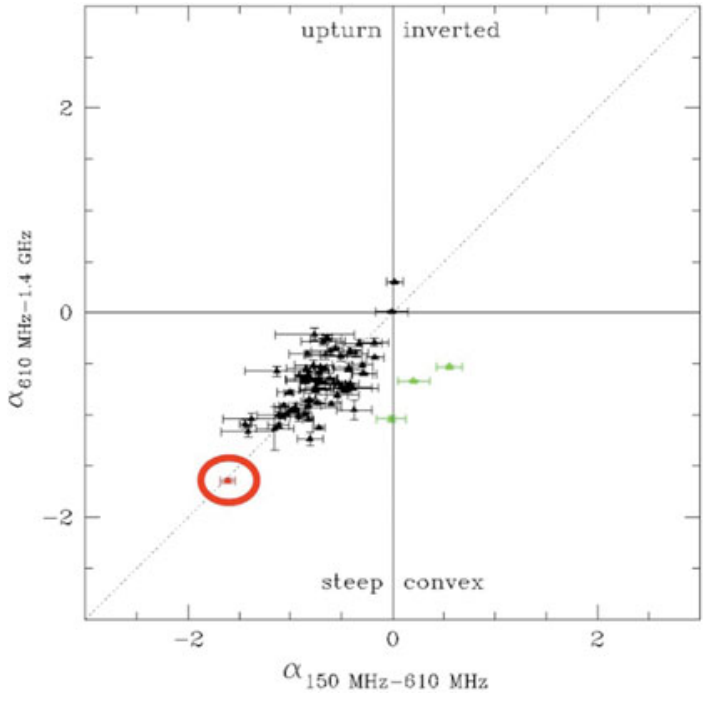

Figure 1. Radio colorcolor plot for 75 sources with $S_{150 \mathrm{MHz}}>15 \mathrm{mJy}: \alpha(610$ $\mathrm{MHz}-1.4 \mathrm{GHz})$ vs. $\alpha(150$ $\mathrm{MHz}$ - $610 \mathrm{MHz}$ (where $\left.S \sim \nu^{\alpha}\right)$. Only three sources show a significant spectral curvature, see green points in the bottom right quadrant).The red circled point indicates the USS source discovered in the field.

\section{HBA LOFAR Commissioning Observations}

The LH was observed with LOFAR (39 core and 9 remote stations) at $150 \mathrm{MHz}$ in October 2011, as part of the commissioning, using a novel observing strategy. One beam was centered on the bright source $3 \mathrm{C} 244.1$, which is located at $\sim 3$ degrees from the center of the Lockman Hole field; a second beam was centered on the Lockman Hole itself. In addition the calibrator 3C196 was separately observed every $30^{\mathrm{min}}$ for one minute. This allowed a good full resolution $(8 " \times 4 ")$ modeling of the 3 C244.1 source, and, as a result, a very good calibration of the entire Lockman Hole field. This ad hoc calibration strategy was applied to 60 of the 120 sub-bands available, yielding very satisfactory results: no major systematic errors are visible in the image, and the noise reached, $1.5 \mathrm{mJy} / \mathrm{b}$, is close to the expected one, $\sim 1 \mathrm{mJy} / \mathrm{b}$. The detailed, high resolution sky model obtained from these observations will be used as a starting point for the calibarion of next observations.

\section{Low frequency Spectral Properties of Faint Radio Sources}

The HBA commisioning data were used to investigate for the first time the source low-frequency spectral properties down to $150 \mathrm{MHz}$ flux densities of the order of $15 \mathrm{mJy}$. The radio color-color diagram obtained using the existing multi-frequency information (including GMRT $610 \mathrm{MHz}$ observations from the literature) is shown in Fig. 1. The radio spectra are generally consistent with single power laws: no significant spectrum curvature is found ( $\langle C\rangle \sim 0$ ), at least down to $150 \mathrm{MHz}$. Very interestingly, an Ultra Steep Spectrum (USS, $\alpha \sim-1.6$ ) source was serendipitely discovered (see red circled dot). This source is likely at high redshift, as it does not have an optical counterpart in the SDSS survey, and is associated with a very faint IR counterpart in SERVS images.

\section{Ongoing Work \& Future Perspectives}

The Lockman Hole was observed with LOFAR in both LBA (10 hrs) and HBA (10 hrs) during LOFAR Cycle 0, as part of the Blank Fields Survey (PI: P. Best). Data reduction is ongoing (Mahony et al., in prep.; van Bemmel et al., in prep.). Additional HBA observations $(6 \times 8$ hours $)$ have been obtained in Cycle 1 . This will allow us to produce the deepest HBA radio image over sky areas of tens of square degrees. 